Check for updates

Cite this: RSC Adv., 2018, 8, 15078

Received 31st January 2018

Accepted 10th April 2018

DOI: $10.1039 / \mathrm{c} 8 \mathrm{ra00952j}$

rsc.li/rsc-advances

\section{Structural and dynamical properties of water adsorption on $\mathrm{PtO}_{2}(001)$}

\begin{abstract}
Yong Yang (iD *ab
The structural, dynamical and electronic properties of water molecules on the $\beta-\mathrm{PtO}_{2}(001)$ surface has been studied using first-principles calculations. For both water monomer and monolayer, the adsorption energies are found to be three to five times larger than that of water adsorption on the Pt surface, and the dissociative adsorption configurations are energetically more stable. The adsorption energies are positively correlated with the charge transfer between the water molecule and the substrate, and the charge-rebalance between the $\mathrm{Pt}$ and $\mathrm{O}$ atoms of $\beta-\mathrm{PtO}_{2}$ upon water adsorption. More interestingly, an exceptionally large redshift is observed in the $\mathrm{OH}$ stretching mode of the adsorbed water monomer, due to the very strong hydrogen bonding with the substrate. The strong water-substrate interactions have significant effects on the molecular orbitals of the chemisorbed water molecules.
\end{abstract}

\section{Introduction}

The adsorption of water molecules on the surfaces of solid state materials is a ubiquitous phenomenon which plays an important role in modifying the surface structure and consequently the stability and reactivity of the surfaces. The presence of water molecules has significant influences on the interactions of surfaces with the other substances. ${ }^{1,2}$ As a product of the oxygen reduction reaction (ORR) that takes place in fuel cells, water naturally presents on the surfaces of the electrode. Platinum $(\mathrm{Pt})$ is the most commonly employed material for the electrode, owing to its high reactivity for catalyzing the ORR. ${ }^{3,4}$ It has been found in previous works ${ }^{5-8}$ that the oxides of Pt can be formed on the Pt surface under oxygen rich conditions or at a potentialimposed interface such as the electrolyte-electrode interface of the proton-exchange membrane fuel cells after some time of application. Recent in situ and real-time experimental measurements ${ }^{9}$ have shown that thin films of Pt oxides which mainly consist of precursor of $\beta-\mathrm{PtO}_{2}$ are formed when the potential is $\sim 1.4 \mathrm{~V}$ with respect to the reversible hydrogen electrode (RHE).

Previous experimental and theoretical works have established that, ${ }^{10,11}$ water molecules are adsorbed molecularly (undissociated) as the wetting layer on Pt surface, with the adsorption energy of $\sim 0.30 \mathrm{eV}$ for water monomer and $\sim 0.52 \mathrm{eV}$ per $\mathrm{H}_{2} \mathrm{O}$ for water hexamer. ${ }^{12}$ To date, however, the adsorption structures of water on the surface of $\mathrm{PtO}_{2}$ remain unknown, which are practically more relevant to the ORR process on the

${ }^{a}$ Key Laboratory of Materials Physics, Institute of Solid State Physics, Chinese Academy of Sciences, Hefei 230031, China.E-mail: wateratnanoscale@hotmail.com;yyang@ theory.issp.ac.cn

${ }^{b}$ University of Science and Technology of China, Hefei 230026, China electrode of fuel cell. In this work, we study the adsorption structures of sub-monolayer and monolayer water molecules on $\beta-\mathrm{PtO}_{2}(001)$ surface. Our first-principles calculations show that, for both water monomer and monolayer adsorption, the molecular adsorption state is found to be energetically less stable than the dissociative adsorption state, in direct contrast to the molecular state of water adsorption on Pt electrode surface. Depending on the adsorption configurations, the adsorption energy of water molecules on $\mathrm{PtO}_{2}(001)$ ranges from $\sim 1.0 \mathrm{eV}$ to $1.7 \mathrm{eV}$, which is three to five times larger than the adsorption of water on Pt surface. The water-substrate interactions have significant effects on the vibrational frequencies and molecular orbitals of the adsorbed water molecules. In particular, an exceptionally large redshift in the frequency of the $\mathrm{O}-\mathrm{H}$ stretching mode of water molecule, $v(\mathrm{OH})$, is observed in the adsorption configuration where a strong hydrogen bond is formed with the substrate. Quantitative analysis show that positive correlation exists between the binding strength of water on $\mathrm{PtO}_{2}(001)$ and the charge transfer from water to the substrate.

The contents of this paper are organized as follows: after this brief introduction, the computational method employed in this study will be described in Sec. 2. The results and discussion regarding the adsorption structures of water molecules on $\beta$ $\mathrm{PtO}_{2}$ (001), the effects of water-substrate interactions on the vibrational properties and electronic structures of the adsorbed water molecules, will be presented in Sec. 3 . The conclusion part is given in Sec. 4 .

\section{Computational methods}

The crystal of $\beta-\mathrm{PtO}_{2}$ has a $\mathrm{CaCl}_{2}$-type structure, which can be synthesized under high pressure conditions. ${ }^{13}$ The experimentally 
determined lattice parameters are as follows: $:^{13} a=4.4839 \AA$ A, $b=$ $4.5385 \AA, c=3.1360 \AA, \alpha=\beta=\gamma=90^{\circ}$ and $Z=2$. The lattice parameters computed using density functional theory (DFT) are the following: $a=4.613 \AA, b=4.556 \AA, c=3.191 \AA, \alpha=\beta=\gamma=$ $90^{\circ}$. As show below, the effects of the small difference between experimental and theoretical lattice parameters are negligible. Therefore, unless specially stated, the experiment lattice parameters will be employed for the simulations. In our study, the $\beta$ $\mathrm{PtO}_{2}(001)$ surface is modeled by separating the $(2 \times 2 \times 3)$ supercell of $\beta-\mathrm{PtO}_{2}$ along the $c$-axis ( $z$-direction) with a vacuum layer of $\sim 10 \AA$ while the $a \& b$-axis extend in the $x y$-plane using periodic boundary conditions. The atomic positions of the bottom three layers of the six-layer slab are fixed to simulate the bulk state. An isolated water molecule is simulated by placing it in a ( $10 \AA \times$ $10 \AA \times 10 \AA$ ) box. The first-principles calculations were carried out by the VASP code, ${ }^{\mathbf{1 4}, 15}$ which is based on DFT. A plane wave basis set and the projector-augmented-wave (PAW) potentials ${ }^{\mathbf{1 6}, 17}$ were employed to describe the electron wave function and the electronion interactions, respectively. The exchange-correlation interactions of electrons are described by the PBE type functional. ${ }^{18}$ The energy cutoff for plane waves is $600 \mathrm{eV}$. With reference to the results obtained using higher energy cutoffs $(700 \mathrm{eV}, 800 \mathrm{eV})$, the energy cutoff of $600 \mathrm{eV}$ ensures the calculated adsorption energies to converge to within an error bar of $\sim 30 \mathrm{meV}$ or better. For structural relaxation and total energy calculations of the $\mathrm{H}_{2} \mathrm{O} / \mathrm{PtO}_{2}$ system, a $2 \times 2 \times 1$ Monkhorst-Pack k-mesh ${ }^{19}$ is generated for sampling the Brillouin zone (BZ). An $8 \times 8 \times 8 \mathrm{k}$-mesh is employed for the calculation of an isolated water molecule.

The adsorption energy ( $\left.E_{\text {ads }}\right)$ of water molecules is calculated via the following formula:

$$
\begin{aligned}
E_{\mathrm{ads}}= & \frac{1}{n}\left\{E\left[\mathrm{PtO}_{2}(001)\right]+n \times E\left[\left(\mathrm{H}_{2} \mathrm{O}\right)_{\text {isolated }}\right]\right. \\
& \left.-E\left[\mathrm{H}_{2} \mathrm{O} / \mathrm{PtO}_{2}(001)\right]\right\}+\Delta E_{\mathrm{ZPV}}
\end{aligned}
$$

where $E\left[\mathrm{H}_{2} \mathrm{O} / \mathrm{PtO}_{2}(001)\right], \quad E\left[\mathrm{PtO}_{2}(001)\right], \quad E\left[\left(\mathrm{H}_{2} \mathrm{O}\right)_{\text {isolated }}\right]$ are respectively the total energies of the adsorption system, the $\mathrm{PtO}_{2}(001)$ substrate, and an isolated water molecule; $n$ is the number of water molecules contained in each simulation supercell. For the simulation of water monomer, $n=1$; and $n=$ 4 for the simulation of 1 monolayer (ML) adsorption, in which the surface unit cell contains four $\mathrm{PtO}_{2}$ units. The term $\Delta E_{\mathrm{ZPV}}$ is the energy correction due to the change of zero-point energy of water molecules, from isolated state to adsorption state. In our calculation, $\Delta E_{\mathrm{ZPV}}$ is computed as follows: $\Delta E_{\mathrm{ZPV}}=\frac{1}{2}\left(\sum_{\mathrm{i}, \text { isolated }} \hbar \omega_{\mathrm{i}}-\sum_{\mathrm{j}, \mathrm{ads}} \hbar \omega_{\mathrm{j}}\right)$, where $\omega_{\mathrm{i}}$ and $\omega_{\mathrm{j}}$ are respectively vibrational frequencies of water molecules at isolated and adsorbed state, and $\hbar$ is the Planck constant. The vibrational frequencies of the water molecules and the adsorption systems were computed using the density functional perturbation theory (DFPT). ${ }^{20,21}$

\section{Results and discussion}

The $\mathrm{PtO}_{2}(001)$ surface on which the water molecules are adsorbed (Fig. 1) has a rectangular surface unit cell, consisting of four line-shape $\mathrm{O}-\mathrm{Pt}-\mathrm{O}$ units which locate at the four vertices of the rectangle. Compared to the Pt atoms of bulk state which are coordinated by six $\mathrm{O}$ atoms, the $\mathrm{Pt}$ atoms on the surface are only coordinated by four $\mathrm{O}$ atoms. Such coordinatively unsaturated atoms serve as the adsorption sites of water molecules. For monomer adsorption, a number of configurations are considered, including the ones that stand upright on or parallel to the top sites of substrate atoms, forming $\mathrm{O}-\mathrm{Pt}$ bonds and/or $\mathrm{H}-\mathrm{O}$ hydrogen bonds. It turns out that the tilted lying configurations that form $\mathrm{O}-\mathrm{Pt}$ bonds with the substrate are energetically favored over the upright standing ones. Three typical configurations, two molecular and one dissociative adsorption structures of water monomers, are schematically depicted in Fig. 1(a-c). The corresponding adsorption energies $\left(E_{\text {ads }}\right)$ and geometric parameters describing the adsorption configurations are tabulated in Table 1 . The values of $E_{\text {ads }}$ are $\sim 1 \mathrm{eV}$ and above, which indicate that water molecules are chemically adsorbed on the $\mathrm{PtO}_{2}(001)$ surface. The adsorption energies ( $\sim 1 \mathrm{eV}$ to $\left.1.7 \mathrm{eV}\right)$ are three to five times that of water adsorption on Pt surface. ${ }^{\mathbf{1 2}}$ In calculations using the theoretical lattice parameters of $\mathrm{PtO}_{2}$, the values of $E_{\text {ads }}$ may differ by several tens of $\operatorname{meV}\left(e . g ., E_{\text {ads }} \sim\right.$ $0.99 \mathrm{eV}$ for the configuration shown in Fig. 1(a), differs by $\sim 30$ $\mathrm{meV}$ ), which is negligible comparing to the magnitude of $E_{\text {ads }}$. For an isolated or free state water molecule, the calculated $\mathrm{HOH}$ angle is $\sim 103.70^{\circ}$ (experimental value: ${ }^{1} 104.52^{\circ}$ ) and the $\mathrm{OH}$ bond length is $\sim 0.972 \AA$ (experimental value: ${ }^{1} 0.957 \AA$ ). Upon adsorption, the $\mathrm{HOH}$ angle is either enlarged (Fig. 1(a)) or contracted (Fig. 1(b) and (c)), and all the $\mathrm{OH}$ bond lengths are elongated. The molecular configuration (Fig. 1(b)) that forms a hydrogen bond with the substrate $\mathrm{O}$ (labeled as $\mathrm{O}^{\mathrm{S}}$ hereafter) is much more stable than the one without hydrogen bonding (Fig. 1(a)). Given that the O-Pt bonds formed between water and the substrate are of similar strength, the strength of the hydrogen bond formed between water and the substrate can therefore be estimated via the difference of adsorption energy, which is $\sim 0.54 \mathrm{eV}$. To our knowledge, this is the strongest hydrogen bond ever found in $\mathrm{OH} \cdots \mathrm{O}$ systems. As shown in Fig. 1(c) and Table 1, the adsorption configuration shown in Fig. 1(b) is further stabilized by breaking the $\mathrm{OH}$ bond that forms hydrogen bond with the substrate and transferring the $\mathrm{H}$ atom to the underlying $\mathrm{O}^{\mathrm{S}}$. As a result, a pair of hydroxyls $(\mathrm{OH})$ which are connected by a hydrogen bond appear on the $\mathrm{PtO}_{2}(001)$ surface.

To get insight into the water-substrate interactions, we have calculated the charge density difference for monomer adsorption, which is defined as follows:

$$
\Delta \rho=\rho\left[\mathrm{H}_{2} \mathrm{O} / \mathrm{PtO}_{2}(001)\right]-\rho\left[\mathrm{PtO}_{2}(001)\right]-\rho\left[\mathrm{H}_{2} \mathrm{O}\right]
$$

where $\rho\left[\mathrm{H}_{2} \mathrm{O} / \mathrm{PtO}_{2}(001)\right], \rho\left[\mathrm{PtO}_{2}(001)\right]$ and $\rho\left[\mathrm{H}_{2} \mathrm{O}\right]$ are the electron densities of the $\mathrm{H}_{2} \mathrm{O} / \mathrm{PtO}_{2}(001)$ system, the $\mathrm{PtO}_{2}(001)$ surface and the water molecule with the same geometry as the adsorption configuration, respectively. The spatial distribution of the charge density difference is shown in Fig. 1(d-f), for the three monomer adsorption configurations studied above. Clearly, stronger water-substrate (measured by $E_{\text {ads }}$ ) interactions are accompanied by larger magnitude of charge 


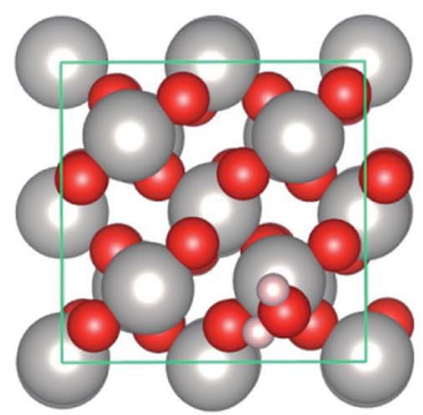

(a)
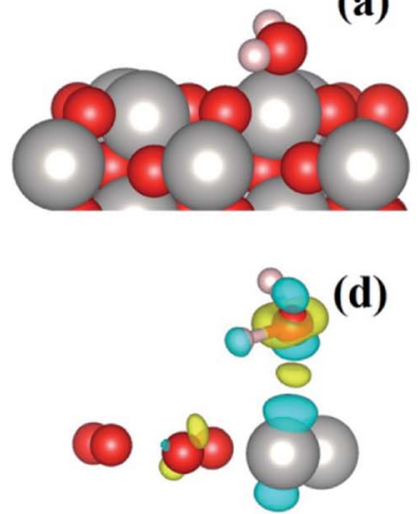

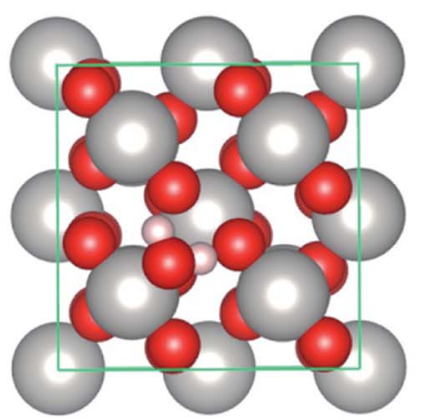

(b)

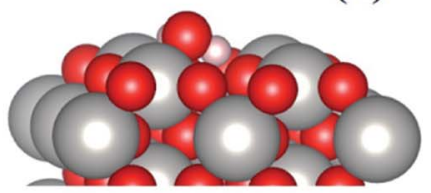

(e)

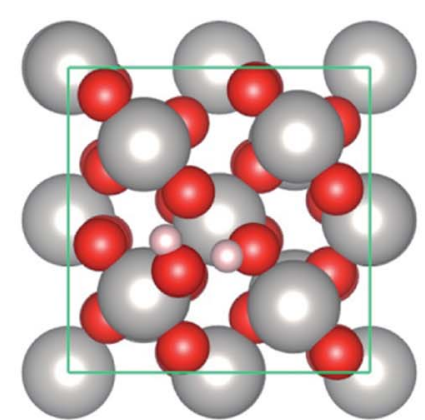

(c)
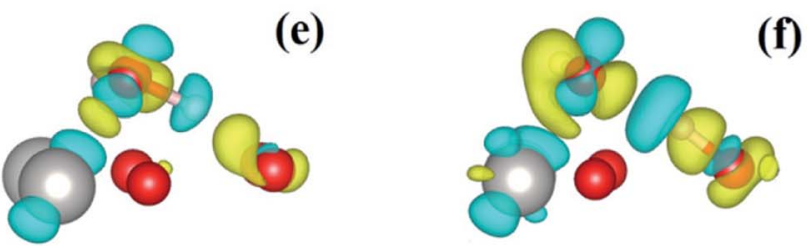

Fig. 1 Panels $(\mathrm{a}-\mathrm{c})$ : top view (upper panels) and side view (lower panels) of the molecular (a and b) and dissociative (c) adsorption of water monomers on $\beta-\mathrm{PtO}_{2}(001)$. Right below the side view of each configuration, is the corresponding charge density difference (panels ( $\left.d-f\right)$ ). The $\mathrm{Pt}$, $\mathrm{O}$, and $\mathrm{H}$ atoms are represented by silver (largest), red (second largest), and white (smallest) spheres, respectively.

redistribution. We tried to make quantitative analysis on the charge transfer between water molecule and the substrate, by performing Bader analysis ${ }^{22,23}$ on the charge densities of the adsorption systems, the $\mathrm{PtO}_{2}(001)$ surface and the water molecule. The net charge transfer, $\Delta q\left(\mathrm{H}_{2} \mathrm{O}\right)$, is defined as $\Delta q\left(\mathrm{H}_{2} \mathrm{O}\right)=$ $q\left(\mathrm{H}_{2} \mathrm{O}\right)_{\text {ads }}-q\left(\mathrm{H}_{2} \mathrm{O}\right)_{\text {isolated }}$, i.e., the change in the total number of electrons after adsorption on $\mathrm{PtO}_{2}(001)$. For the three adsorption configurations shown in Fig. $1(\mathrm{a}-\mathrm{c})$, the value of $\Delta q\left(\mathrm{H}_{2} \mathrm{O}\right)$ is calculated to be $\sim-0.150 e,-0.153 e$, and $-0.571 e$, respectively. We have also analyzed the variation of the total number of electrons associated with the substrate $\mathrm{O}$ and $\mathrm{Pt}$, and found that $\Delta q\left(\mathrm{O}^{\mathrm{S}}\right)$ is $\sim 0.164 e, 0.273 e$, and $0.761 e$, and $\Delta q(\mathrm{Pt})$ is $\sim-0.016 e$, $-0.121 e$, and $-0.191 e$ for the three configurations, respectively. It is clear that electrons are transferred from the water molecule (precisely, the $\mathrm{H}$ atoms of adsorbed water molecule) to the substrate $\mathrm{O}$ atoms. Moreover, the charges around the underlying Pt are also rebalanced by donating a small amount of electrons to the neighboring substrate $\mathrm{O}$. Note that the small deviation from the charge-neutral condition $\left(\Delta q\left(\mathrm{H}_{2} \mathrm{O}\right)+\Delta q\left(\mathrm{O}^{\mathrm{S}}\right)\right.$ $+\Delta q(\mathrm{Pt})=0)$ is simply due to numerical error. Nevertheless, the results demonstrate unambiguously that the water-substrate interactions are enhanced by charge transfer: larger $E_{\text {ads }}$ is associated with larger $\Delta q\left(\mathrm{O}^{\mathrm{S}}\right)$.

In the case of water monolayer (ML) adsorption, two configurations are considered (Fig. 2): the molecular and dissociative type, in which the water molecules have similar geometries as the monomers shown in Fig. 1(b) and (c). As seen from Table 1, the $1 \mathrm{ML}$ molecular adsorption has the same $E_{\text {ads }}$ as the monomer configuration shown in Fig. 1(b), while the $1 \mathrm{ML}$ dissociative adsorption has a value of $E_{\text {ads }}$ slightly smaller

Table 1 The adsorption energies and geometric parameters for the monomer and 1 monolayer (ML) water adsorption on $\beta-\mathrm{PtO} 2$ (001). For $1 \mathrm{ML}$ adsorption, the averaged geometric parameters are listed with the standard derivation provided in the parentheses. $\angle \mathrm{HOH}$ : the $\mathrm{HOH}$ angle; $\mathrm{OH}{ }^{1}$ and $\mathrm{OH}^{2}$ : the length of the two $\mathrm{OH}$ bonds; $\Delta \mathrm{O}_{x y}$ : the lateral displacement of the adsorbed $\mathrm{O}$ away from the precise top of Pt; $\Delta \mathrm{O}_{z}$ : the vertical distance of the adsorbed $\mathrm{O}$ from the precise top of $\mathrm{Pt}$; $\mathrm{O}^{\mathrm{A}}-\mathrm{Pt}$ : the distance between the adsorbed $\mathrm{O}$ and $\mathrm{Pt}$; $\mathrm{H}^{\mathrm{A}}-\mathrm{O}^{\mathrm{S}}$ : the nearest distance between the $\mathrm{H}$ of adsorbed $\mathrm{H}_{2} \mathrm{O}$ and substrate $\mathrm{O}$

\begin{tabular}{|c|c|c|c|c|c|c|c|c|}
\hline Configuration & $\begin{array}{l}E_{\text {ads }}(\mathrm{eV} \\
\left.\text { per } \mathrm{H}_{2} \mathrm{O}\right)\end{array}$ & $\angle \mathrm{HOH}\left(^{\circ}\right)$ & $\mathrm{OH}^{1}(\AA)$ & $\mathrm{OH}^{2}(\AA)$ & $\Delta \mathrm{O}_{x y}(\AA)$ & $\Delta \mathrm{O}_{z}(\AA)$ & $\mathrm{O}^{\mathrm{A}}-\mathrm{Pt}(\AA)$ & $\mathrm{H}^{\mathrm{A}}-\mathrm{O}^{\mathrm{S}}(\AA)$ \\
\hline Fig. 1(a) & 1.02 & 106.32 & 0.996 & 0.978 & 0.928 & 1.955 & 2.164 & 1.915 \\
\hline Fig. 1(c) & 1.71 & 99.60 & 1.718 & 0.977 & 1.226 & 1.575 & 1.996 & 1.017 \\
\hline Fig. 2(a) & 1.56 & $97.14(0.04)$ & $1.058(0.001)$ & $0.993(0.001)$ & $1.411(0.000)$ & $1.534(0.000)$ & $2.084(0.000)$ & $1.519(0.001)$ \\
\hline Fig. 2(b) & 1.66 & $96.99(0.03)$ & $1.673(0.000)$ & $0.981(0.001)$ & $1.262(0.000)$ & $1.544(0.000)$ & $1.995(0.000)$ & $1.024(0.000)$ \\
\hline
\end{tabular}


(differs by $\sim 0.05 \mathrm{eV}$ ) than the dissociative monomer shown in Fig. 1(c). This is understandable when considering the following facts: the adsorption geometries of each water molecule of the $1 \mathrm{ML}$ molecular configuration (Fig. 2(a)), such as the $\mathrm{HOH}$ angles, $\mathrm{OH}$ bond lengths, the positional displacement of the $\mathrm{O}$ atom in water molecule from the $\mathrm{Pt}$ top site, and the lengths of hydrogen bonds, are nearly identical to that of the monomer in Fig. 1(b); there are, however, minor differences between the geometric parameters of the $1 \mathrm{ML}$ dissociative configuration (Fig. 2(b)) and that of the monomer dissociative adsorption in Fig. 1(c). On the other hand, for $1 \mathrm{ML}$ adsorption, either molecular or dissociative, each constituent water molecule shares nearly identical adsorption geometries (Fig. 2 and Table 1). Due to the very strong water-substrate interactions (the order of $1 \mathrm{eV}$ and above), the water-water interactions, i.e., the hydrogen bonding between water molecules, consequently plays a minor role in determining the adsorption structures for monolayer and submonolayer coverages.

The strong water-substrate interactions have significant effects on the adsorption structures of water molecules and therefore the vibrational properties and electronic structures. Shown in Fig. 3, are the three normal modes of vibration of an isolated water molecule, together with the corresponding normal modes of vibration of the adsorbed water monomers on $\mathrm{PtO}_{2}(001)$, in the order of vibrational frequencies. For an isolated water molecule, the asymmetric $\mathrm{OH}$ stretching mode $\left(\nu_{\mathrm{a}}(\mathrm{OH})\right)$ has the highest frequency $\left(\tilde{v}_{1}=3842 \mathrm{~cm}^{-1}\right)$, the symmetric $\mathrm{OH}$ stretching mode $\left(\nu_{\mathrm{s}}(\mathrm{OH})\right)$ is second highest $\left(\tilde{v}_{2}=\right.$ $\left.3737 \mathrm{~cm}^{-1}\right)$, and the $\mathrm{HOH}$ bending mode $(\delta(\mathrm{HOH}))$ is the third $\left(\tilde{v}_{3}=1594 \mathrm{~cm}^{-1}\right.$ ); all of which compare well (within an error bar of $\sim 2 \%)$ with the data reported in literatures $\left(\tilde{v}_{1}=3756 \mathrm{~cm}^{-1}\right.$; $\left.\tilde{v}_{2}=3657 \mathrm{~cm}^{-1} ; \tilde{v}_{3}=1595 \mathrm{~cm}^{-1}\right) .{ }^{1,24}$

For the molecular adsorption of water monomer, significant redshifts of vibrational frequencies are found in both the asymmetric and symmetric $\mathrm{OH}$ stretching mode, while only minor changes are observed in the $\mathrm{HOH}$ bending mode (Fig. 3(d-i)). Firstly, the magnitude of redshift in the $\mathrm{OH}$ stretching modes (asymmetric or symmetric) of the molecular configurations, $\Delta \nu(\mathrm{OH})$, is found to increases with $E_{\text {ads }}$, and the absolute value of charge transfer $\Delta q$. Secondly, the order of $\Delta \nu(\mathrm{OH})$, can also be measured by the $\mathrm{OH}$ bond lengths (Table 1 ): for the same mode, longer $\mathrm{OH}$ bond lengths implies smaller vibrational frequency, or larger redshift $\Delta \nu(\mathrm{OH})$. In addition, smaller redshift is observed in the asymmetric $\mathrm{OH}$ stretching mode of the same monomer configuration, i.e., $\Delta \nu_{\mathrm{a}}(\mathrm{OH})<\Delta \nu_{\mathrm{s}}(\mathrm{OH})$. The HOH bending/ scissoring motions are least affected by the adsorption geometry which is confined on the $\mathrm{PtO}_{2}(001)$ plane. Therefore, much smaller redshift is found in the bending mode.

Returning to the redshift of the $\mathrm{OH}$ stretching modes, we find that an exceptionally large redshift presents in the symmetric stretching mode of the water monomer shown in Fig. 1(b), with a vibrational frequency of $\tilde{v}_{2}=2224 \mathrm{~cm}^{-1}$, which corresponds to a redshift $\Delta \nu_{\mathrm{s}}(\mathrm{OH})=1513 \mathrm{~cm}^{-1}$. To the best of our knowledge, this is the largest redshift of $\mathrm{OH}$ stretching mode ever reported for the water-based systems, in which the redshift of $\nu(\mathrm{OH})$ due to hydrogen bonds usually ranges from tens to several hundred $\mathrm{cm}^{-1}$ and typically $\sim 500 \mathrm{~cm}^{-1} \cdot .^{25-27}$ The giant redshift can be explained by the

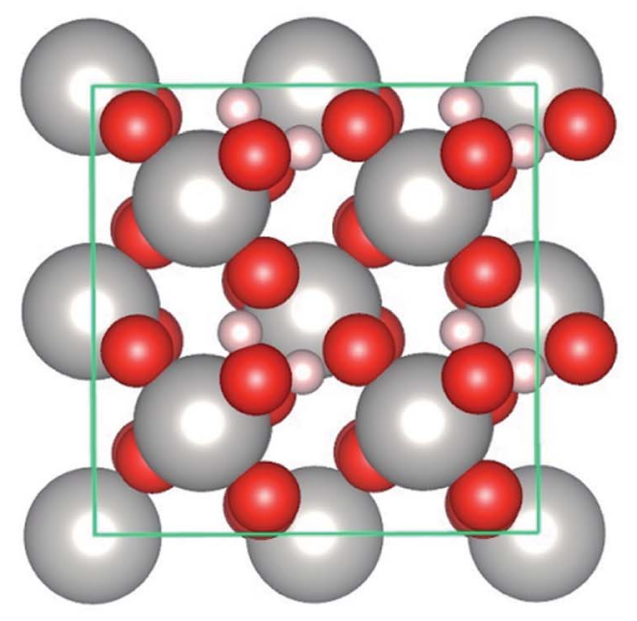

(a)

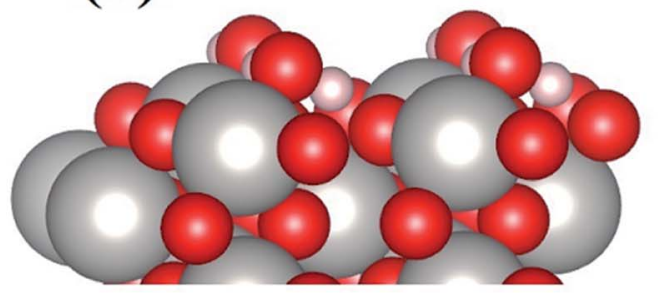

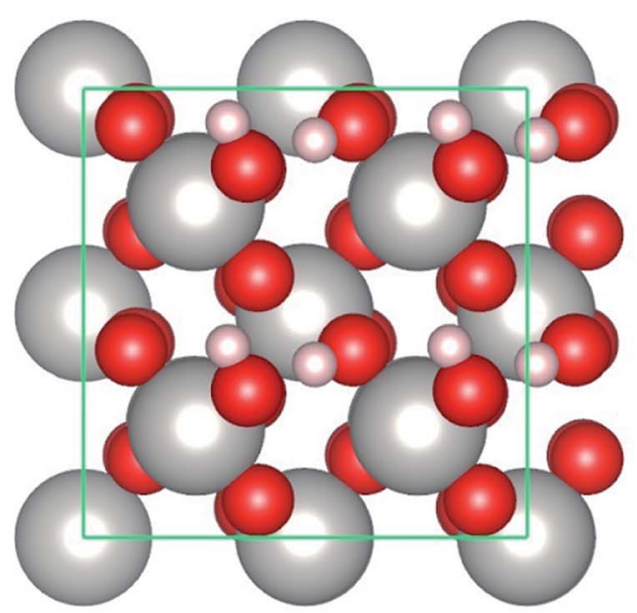

(b)

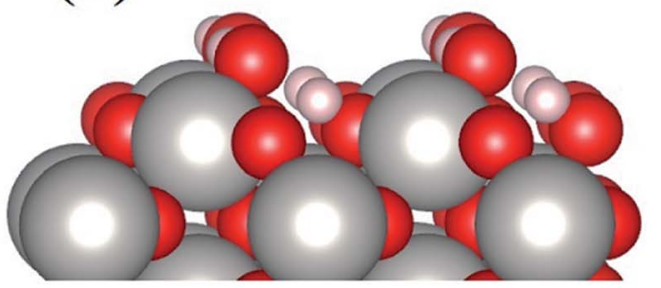

Fig. 2 Molecular (a) and dissociative (b) adsorption of $1 \mathrm{ML}$ water on $\beta-\mathrm{PtO}_{2}(001)$. 

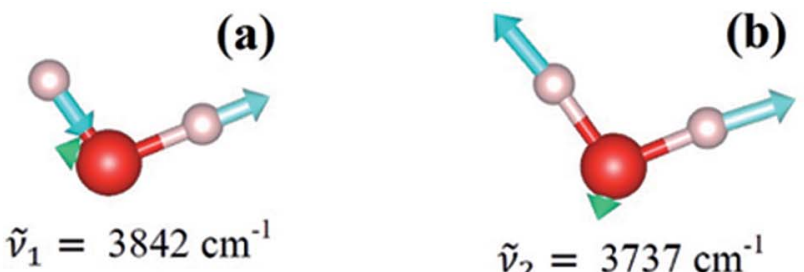

$\tilde{v}_{2}=3737 \mathrm{~cm}^{-1}$

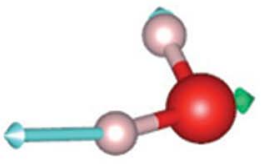

(e)

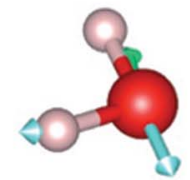

(d)

$$
\tilde{v}_{1}=3704 \mathrm{~cm}^{-1}
$$

$\overline{\mathrm{PtO}_{2} \text { (001) }}$

(g)

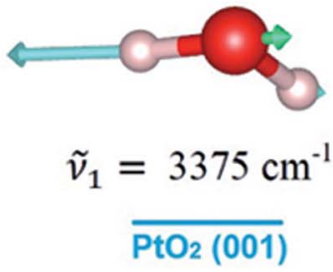

(j)

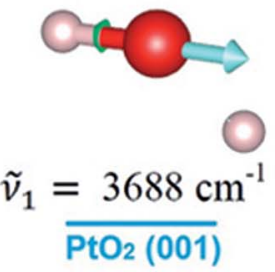

$\tilde{v}_{2}=3329 \mathrm{~cm}^{-1}$

$\overline{\mathrm{PtO}_{2} \text { (001) }}$

(h)

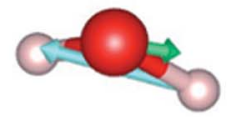

$\tilde{v}_{2}=2224 \mathrm{~cm}^{-1}$

$\overline{\mathrm{PtO}_{2}(001)}$

(k)

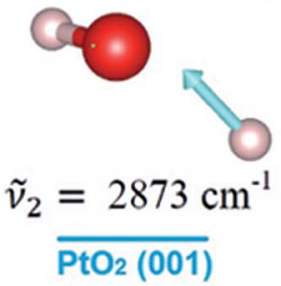

(c)

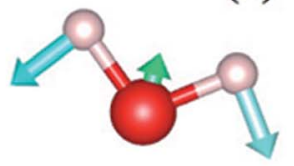

$\tilde{v}_{3}=1594 \mathrm{~cm}^{-1}$

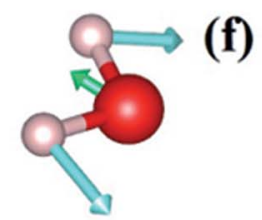

$\tilde{v}_{3}=1552 \mathrm{~cm}^{-1}$

$\overline{\mathrm{PtO}_{2}(001)}$

(i)

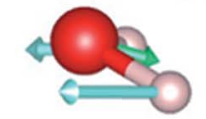

$\frac{\tilde{v}_{3}=1556 \mathrm{~cm}^{-1}}{\mathrm{PtO}_{2}(001)}$

Fig. 3 From top to bottom, the first three normal modes of vibration of an isolated water molecule (panels (a-c)), and the water monomers on $\beta-\mathrm{PtO}_{2}(001)$ as shown in Fig. 1(a) (panels (d-f)), Fig. 1(b) (panels ( $\left.\mathrm{g}-\mathrm{i}\right)$ ), and Fig. 1(c) (panels $(j-\mathrm{l})$ ), respectively.

very strong hydrogen bond formed between the adsorbed water molecule and the substrate $\mathrm{O}$. As discussed above, the strength of the hydrogen bond is estimated to be $\sim 0.54 \mathrm{eV}$, being probably the strongest in the $\mathrm{OH} \cdots \mathrm{O}$ systems. The length of the related $\mathrm{OH}$ bond is elongated to be $\sim 1.058 \AA$, increased by $0.086 \AA$ with comparison to the isolated one. The remarkable weakening of $\mathrm{OH}$ bond leads to the giant redshift in the $\nu(\mathrm{OH})$ mode.

In the case of dissociative adsorption, a pair of $\mathrm{OH}$ groups presents (Fig. 1(c) and (f)): the dangling $\mathrm{OH}$ from the adsorbed water molecule, and the new $\mathrm{OH}$ group formed by the transferred $\mathrm{H}$ and substrate $\mathrm{O}^{\mathrm{S}}$. By comparing the frequency of their $\mathrm{OH}$ stretching modes (Fig. $3(\mathrm{j})$ and (k)), i.e., $3688 \mathrm{~cm}^{-1}$ versus $2873 \mathrm{~cm}^{-1}$, a large redshift in $\nu(\mathrm{OH})$ is observed, which is
$\Delta \nu(\mathrm{OH})=815 \mathrm{~cm}^{-1}$. Such a large redshift in the $\mathrm{OH}$ stretching is again due to the strong hydrogen bonding interactions between the dangling $\mathrm{OH}$ and the newly formed $\mathrm{OH}$ on the substrate, which can be schematically denoted as $\mathrm{O} \cdots \mathrm{HO}^{\mathrm{S}}$. Another feature characterized by the vibration mode is the absence of $\mathrm{HOH}$ bending mode, which is typically $\sim 1500 \mathrm{~cm}^{-1}$ for the molecularly adsorbed states (Fig. 3(f) and (i)). Instead, one sees a vibration mode relating with the bending motion of the $\mathrm{HO}^{\mathrm{S}}$ group on the $\mathrm{PtO}_{2}(001)$ surface (Fig. 3(1)), with a frequency of $\tilde{v}_{3}=1255 \mathrm{~cm}^{-1}$.

Fig. 4(a) shows the calculated electronic density of states (DOS) of an isolated water molecule. From left (deep level) to right (shallow level), the four discrete peaks/energy levels of valence electrons correspond to the so-called molecular orbitals 
(MOs) named as $2 a_{1}, 1 b_{2}, 3 a_{1}$, and $1 b_{1}$, respectively. In the picture of linear combination of atomic orbitals (LCAO), the $2 \mathrm{a}_{1}$ and $3 \mathrm{a}_{1}$ MOs mainly consist of the 1 s orbitals of the two $\mathrm{H}$ atoms, the $2 \mathrm{~s}$ and $2 \mathrm{p}$ orbitals of the $\mathrm{O}$ atom; the $1 \mathrm{~b}_{2} \mathrm{MO}$ comprises mainly of the 1 s orbitals of $\mathrm{H}$ and the $2 \mathrm{p}$ orbitals of $\mathrm{O}$; and finally $1 \mathrm{~b}_{1}$, the highest occupied molecular orbital (HOMO), consists mainly of the $2 \mathrm{p}$ orbitals of $\mathrm{O}$, which is usually called the "lone pair" of electrons.

To explore the effects of water-substrate interactions on the MOs, the site projected DOS (PDOS) of the water monomers on $\mathrm{PtO}_{2}(001)$ are displayed in Fig. 4(b-d), for the molecular and dissociative configurations. Hereafter, the molecular adsorption configuration depicted in Fig. 1(a) is indicated as configuration 1 (abbr.: cfg.1), and that in Fig. 1(b) as configuration 2 (abbr.: cfg.2), for the simplicity of discussion. As a consequence of the strong water-substrate interactions, the energy levels near the Fermi level are broadened and the two MOs $3 a_{1}$ and $1 b_{1}$ overlaps with each other while the $2 a_{1}$ and $1 b_{2}$ orbitals remain untouched in molecular cfg.1. The deeper MO $1 b_{2}$ overlaps slightly with $3 \mathrm{a}_{1}$ and $1 \mathrm{~b}_{1}$ in molecular cfg. 2 , where the binding with substrate is stronger. In the case of dissociative adsorption where the strongest water-substrate interactions present, the three MOs $1 b_{2}, 3 a_{1}$ and $1 b_{1}$ are broadened and deeply mixed with each other (Fig. 4(d)). In addition, the deepest valence MO $2 \mathrm{a}_{1}$ is also perturbed and modified.

We go further to study the influence of water-substrate interactions on the wave functions of the MOs $\left(\psi_{\mathrm{MO}}\right)$, by investigating the spatial distribution of the charge densities, $\left|\psi_{\mathrm{MO}}\right|^{2}$, as plotted in Fig. 5, for the isolated water molecule (Fig. 5(a)) and the adsorbed ones (Fig. 5(b-d)). The charge density of the MOs of an isolated water molecule is simply the partial charge density of each valence level as shown in Fig. 4(a). For the MOs of an adsorbed water monomer, the corresponding charge density is obtained by subtracting the partial charge density of the substrate $\mathrm{PtO}_{2}$ from the whole $\mathrm{H}_{2} \mathrm{O} / \mathrm{PtO}_{2}$ system within a specified energy interval as indicated by the PDOS shown in Fig. 4(b-d). The Im-character (s, p, ...) of the MOs can be obtained by projecting the wave functions onto the spherical harmonics. The results are displayed in Table 2, which describe the major characteristics of the wave functions contributed from the atomic orbitals. As expected, the MOs $\left(3 a_{1}, 1 b_{1}\right)$ near the Fermi level are mostly affected and the $2 \mathrm{a}_{1}$ orbital which locates far away from the Fermi level is the least perturbed with comparison to the isolated molecule. Aside from the variations in the occupation numbers of the $\mathrm{s}$ and $\mathrm{p}_{x}, \mathrm{p}_{y}, \mathrm{p}_{z}$-orbitals, which are partly due to the rotation of coordination systems where the spherical harmonics are represented, only minor changes are found in the sum of the $l m$-components of the $1 b_{1}$ orbitals of the molecularly adsorbed monomers.

By contrast, one sees significant decrease in the sum of the lm-components of the $1 b_{1}$ orbital of the dissociative water monomer. Such changes may be attributed to the mixing of the MOs, and the intrinsic incompleteness of the atomic orbitals in expanding the MOs' wave functions. Owing to the strong watersubstrate interactions, the former MOs near the Fermi level are deeply mixed with each other and form new MOs, which we
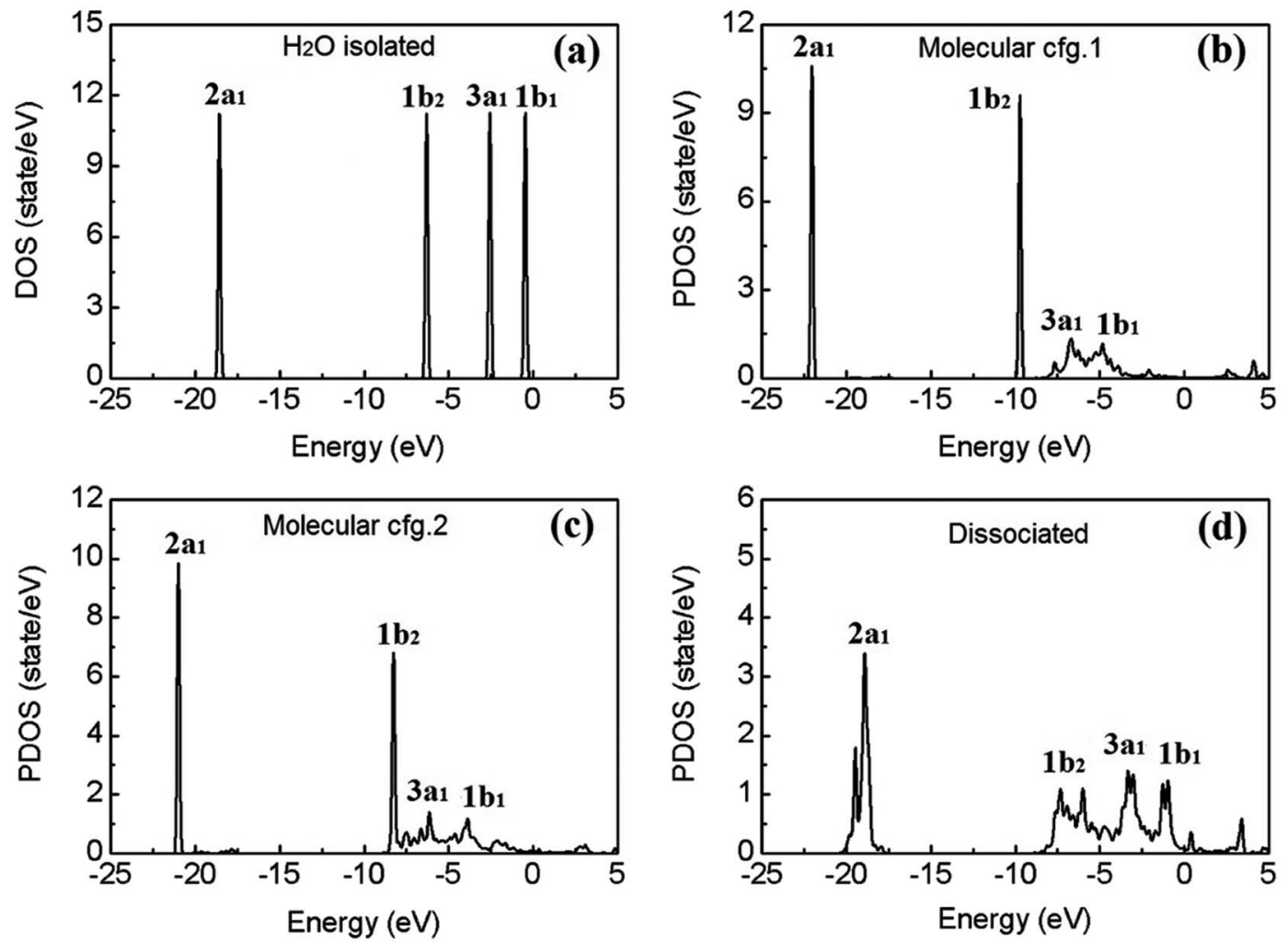

Fig. 4 Calculated electron density of states (DOS) of an isolated water molecule (a), and the site-projected DOS (PDOS) of the molecular (b and c) and dissociated (d) water monomers on $\beta-\mathrm{PtO}_{2}(001)$. The Fermi level of the system is set at $0 \mathrm{eV}$. 

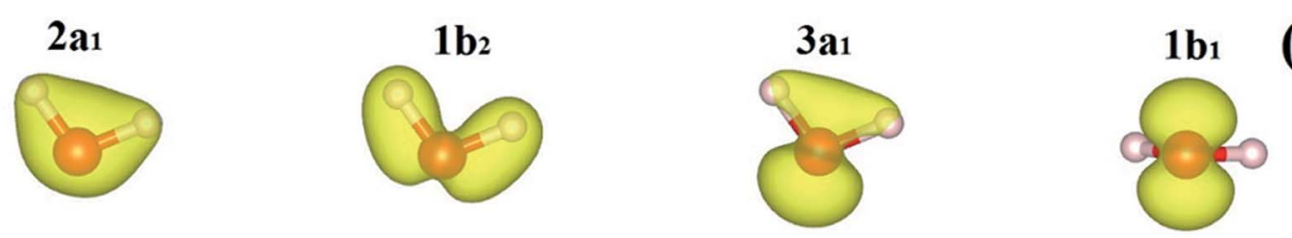

(a)
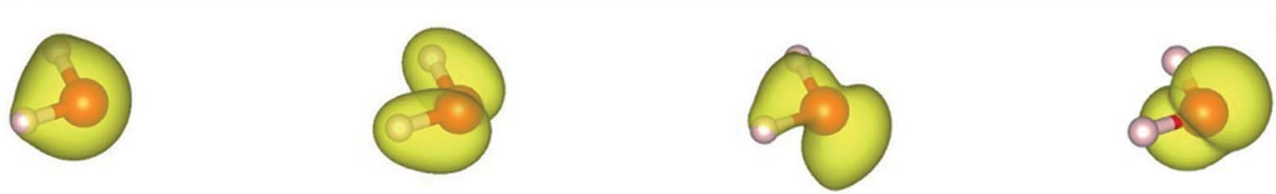

(b)
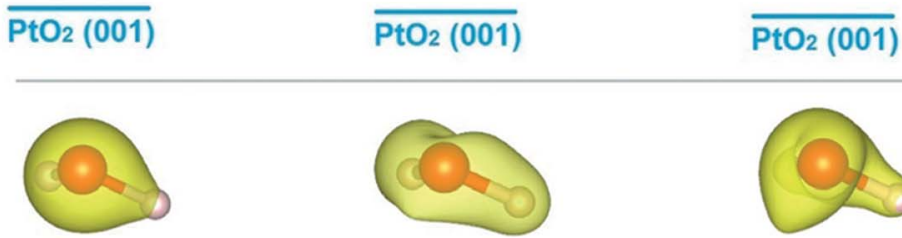

$\overline{\mathrm{PtO}_{2}(001)}$

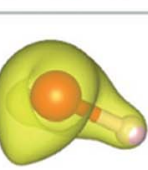

$\overline{\mathrm{PtO}_{2}(001)}$

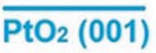

$\overline{\mathrm{PtO}_{2}(001)}$

$\overline{\mathrm{PtO}_{2}(001)}$
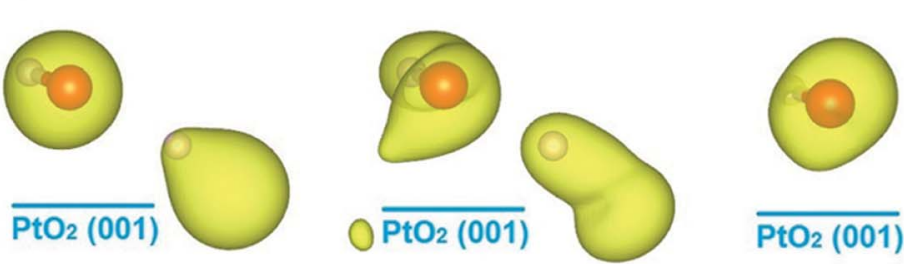

(d)

Fig. 5 Isosurfaces of the charge densities of the molecular orbitals of water molecules, $\left|\psi_{\mathrm{MO}}\right|^{2}$, as identified in Fig. 4, for the isolated state (a), and the molecular cfg.1 (b), cfg.2 (c), and dissociative (d) monomers on $\beta-\mathrm{PtO}_{2}(001)$. The isovalue for charge density plotting is $0.035 e /(\mathrm{Bohr})^{3}$.

provisionally name as " $3 a_{1}+1 b_{1}$ " for molecular cfg. 1 and cfg. 2 , and " $1 b_{2}+3 a_{1}+1 b_{1}$ " for the dissociated one. Within the new MO with modified PDOS, gapless transition between the neighboring energy levels can happen. From the data listed in Table 2, the sum of $l m$-components of the new MO $3 \mathrm{a}_{1}+1 \mathrm{~b}_{1}$ is 1.377 and 1.485 for molecular cfg. 1 and cfg.2, and is 2.123 for

Table 2 Calculated $l m$-components of the wave functions of the water molecular orbitals (MO) of the isolated state, molecular and dissociative adsorption on $\beta-\mathrm{PtO}_{2}(001)$ surface. The abbreviation "cfg" represents "configuration". The sum of the $l m$-components of each $\mathrm{MO}$, Im-sum, is also presented

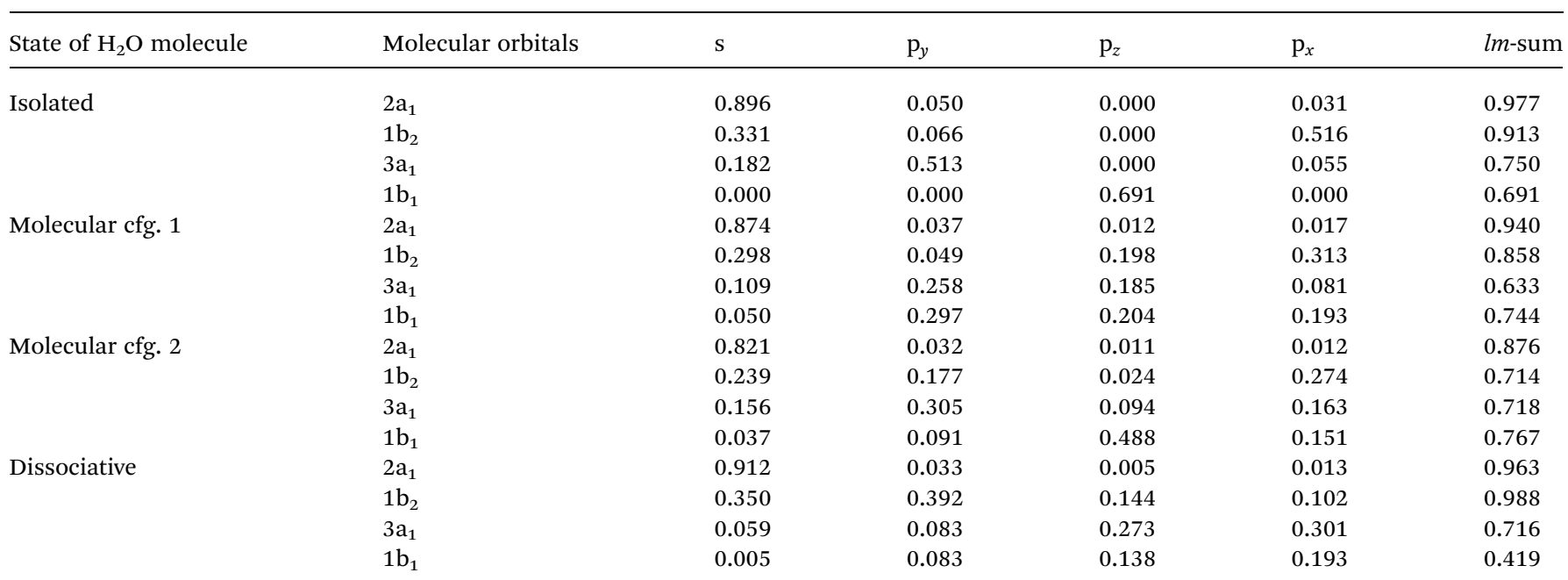


the new MO $1 b_{2}+3 a_{1}+1 b_{1}$. On average, the sum of lmcomponents for one orbital is $1.377 / 2,1.485 / 2$, and $2.123 / 3$, $\sim 70 \%$ of the full occupation, which is 1 by considering the normalization of electron wave function. Similar situation is found in describing the HOMO of an isolated water molecule (Table 2). This originates from the fact that the sp-orbitals projected on the waver functions of the MOs are not a complete basis set and therefore inevitably miss some features. Based on the analysis above and the data in Table 2, one can find that the energy center of HOMO is pushed down due to the mixing of the MOs near Fermi level and the formation of new MOs. From the point of energy, such down-shift of the occupied energy levels of electrons helps further stabilize the adsorption configuration.

\section{Conclusion}

To summarize, we study the adsorption of water molecules on $\beta$ $\mathrm{PtO}_{2}(001)$ surface using DFT calculations. It is found that both monomer and monolayer water molecules are chemically adsorbed, with much larger adsorption energies than the adsorption on $\mathrm{Pt}$, and the dissociative configurations being energetically favored, which is different from the case of water on Pt surface. Detailed analysis reveals that, the strength of water-substrate interactions are positively correlated with the magnitude of water-substrate charge transfer, and the chargerebalance between the substrate $\mathrm{Pt}$ and $\mathrm{O}$ atoms. Due to the very strong hydrogen bonding interactions between the molecularly adsorbed water monomer and the $\mathrm{PtO}_{2}(001)$, a giant redshift in the vibrational frequency of the $\mathrm{OH}$ stretching mode is observed. The strong water-substrate interactions also have significant effects on the electronic structures of the adsorbed molecules, in which the energy levels near the Fermi level are broadened and the molecular orbitals are deeply mixed. We expect that results presented here can be tested by traditional vibrational spectroscopies (e.g., Raman and infrared), ${ }^{1}$ ultraviolet photoemission spectroscopy measurements, as well as the recently developed scanning tunneling microscopy which is capable of imaging the molecular orbitals of water with subatomic resolution. ${ }^{28}$ The adsorption of multilayer water molecules on the $\mathrm{PtO}_{2}$ (001) surface, and their effects on the ORR in fuel cell applications will be the subject of future research.

\section{Conflicts of interest}

The author declares no conflicts of interest.

\section{Acknowledgements}

This work is supported by the National Natural Science Foundation of China (No. 11474285). I gratefully acknowledge the support from the staff of the Hefei Branch of Supercomputing Center of Chinese Academy of Sciences, and the crew of Center for Computational Materials Science of the Institute for Materials Research, Tohoku University for their continuous support of the SR16000 supercomputing facilities.

\section{References}

1 P. A. Thiel and T. E. Madey, Surf. Sci. Rep., 1987, 7, 211-385.

2 M. A. Henderson, Surf. Sci. Rep., 2002, 46, 1-308.

3 J. K. Nørskov, J. Rossmeisl, A. Logadottir, L. Lindqvist, J. R. Kitchin, T. Bligaard and H. Jónsson, J. Phys. Chem. B, 2004, 108, 17886-17892.

4 J. Greeley, I. E. L. Stephens, A. S. Bondarenko, T. P. Johansson, H. A. Hansen, T. F. Jaramillo, J. Rossmeisl, I. Chorkendorff and J. K. Nørskov, Nat. Chem., 2009, 1, 552.

5 C. R. Parkinson, M. Walker and C. F. McConville, Surf. Sci., 2003, 545, 19.

6 W. X. Li, L. Österlund, E. K. Vestergaard, R. T. Vang, J. Matthiesen, T. M. Pedersen, E. Lægsgaard, B. Hammer and F. Besenbacher, Phys. Rev. Lett., 2004, 93, 146104.

7 J. G. Wang, W. X. Li, M. Borg, J. Gustafson, A. Mikkelsen, T. M. Pedersen, E. Lundgren, J. Weissenrieder, J. Klikovits, M. Schmid, B. Hammer and J. N. Andersen, Phys. Rev. Lett., 2005, 95, 256102.

8 Y. Liu, M. Mathias and J. Zhang, Electrochem. Solid-State Lett., 2010, 13, B1.

9 H. Imai, K. Izumi, M. Matsumoto, Y. Kubo, K. Kato and Y. Imai, J. Am. Chem. Soc., 2009, 131, 6293.

10 H. Ogasawara, B. Brena, D. Nordlund, M. Nyberg, A. Pelmenschikov, L. G. M. Pettersson and A. Nilsson, Phys. Rev. Lett., 2002, 89, 276102.

11 S. Meng, L. F. Xu, E. G. Wang and S. Gao, Phys. Rev. Lett., 2002, 89, 176104.

12 S. Meng, E. G. Wang and S. Gao, Phys. Rev. B: Condens. Matter Mater. Phys., 2004, 69, 195404.

13 K.-J. Range, F. Rau, U. Klement and A. M. Heyns, Mater. Res. Bull., 1987, 22, 1541.

14 G. Kresse and J. Hafner, Phys. Rev. B: Condens. Matter Mater. Phys., 1993, 47, 558.

15 G. Kresse and J. Furthmüller, Phys. Rev. B: Condens. Matter Mater. Phys., 1996, 54, 11169.

16 P. E. Blöchl, Phys. Rev. B: Condens. Matter Mater. Phys., 1994, 50, 17953.

17 G. Kresse and D. Joubert, Phys. Rev. B: Condens. Matter Mater. Phys., 1999, 59, 1758.

18 J. P. Perdew, K. Burke and M. Ernzerhof, Phys. Rev. Lett., 1996, 77, 3865.

19 H. J. Monkhorst and J. D. Pack, Phys. Rev. B: Solid State, 1976, 13, 5188.

20 S. Baroni and R. Resta, Phys. Rev. B: Condens. Matter Mater. Phys., 1986, 33, 7017.

21 M. Gajdoš, K. Hummer, G. Kresse, J. Furthmüller and F. Bechstedt, Phys. Rev. B: Condens. Matter Mater. Phys., 2006, 73, 045112.

22 R. Bader, Atoms in Molecules: A Quantum Theory, Oxford University Press, New York, 1990.

23 G. Henkelman, A. Arnaldsson and H. Jónsson, Comput. Mater. Sci., 2006, 36, 354-360.

24 D. Eisenberg and W. Kauzmann, The Structure and Properties of Water, Oxford University Press, New York, 1969. 
25 M. S. Bergen, D. Schuh, M. G. Sceats and S. A. Rice, J. Chem. Phys., 1978, 69, 3477.

26 F. Huisken, M. Kaloudis and A. Kulcke, J. Chem. Phys., 1996, 104, 17.
27 C. J. Burnham, G. F. Reiter, J. Mayers, T. Abdul-Redah, H. Reichert and H. Dosch, Phys. Chem. Chem. Phys., 2006, 8, 3966.

28 J. Guo, X. Meng, J. Chen, J. Peng, J. Sheng, X.-Z. Li, L. Xu, J.-R. Shi, E. G. Wang and Y. Jiang, Nat. Mater., 2014, 13, 184. 